\title{
On the bond between the chemical reaction rate and the velocity of the system under study
}

\author{
E. V. Veitsman \\ Veitsman's Science Project, 28 room, 5 Klimashkin Str., 123557, Moscow, Russia
}

Email address:

ev_veitsman@mail.ru (E. V. Veitsman)

To cite this article:

E. V. Veitsman. On the Bond between the Chemical Reaction Rate and the Velocity of the System under Study, American Journal of Modern Physics. Vol. 2, No. 2, 2013, pp. 93-97. doi: 10.11648/j.ajmp.20130202.21

\begin{abstract}
On the basis of special relativity and irreversible thermodynamics it is shown how the chemical reaction rates vary under relativistic conditions. It is shown as well that the absolute temperature $T$ will vary according to X.Ott under these conditions. It can be represented as the trace of the tensor of rank 2.
\end{abstract}

Keywords: Chemical Reaction Rates, Temperature, Relativistic Conditions

\section{Introduction}

The problems of chemical reaction rates as $v \rightarrow c$ were not almost studied until the present. Of course, today these problems have exclusively academic importance, but having solved them, first, we deepen our knowledge about chemical reaction rates, and, second, we may finally solve a conflict taking place in relativistic thermodynamics which (the conflict) we already tried to solve earlier (E.Veitsman ${ }^{1}$ ).

Let us have a chemical reaction, e.g., of the type

$$
v \mathrm{~A} \Leftrightarrow v \mathrm{~B}+\mathrm{Q}
$$

for example,

$$
\mathrm{O}_{2} \Leftrightarrow 2 \mathrm{O}+\mathrm{Q}
$$

where $v_{\mathrm{A}}$ and $v_{\mathrm{B}}$ are stoichiometric coefficients of substances A and B in the reaction (1), Q is a chemical reaction heat $(\mathrm{J})$.

Then the reaction rate $w(\mathrm{~mol} / \mathrm{s})$ will be at $v<<_{c}$ :

$$
w=-\frac{1}{v_{\mathrm{A}}} \frac{d[\mathrm{~A}]}{d t}=\frac{1}{v_{\mathrm{B}}} \frac{d[\mathrm{~B}]}{d t}
$$

where $t$ is the time, and [A] and [B] are the numbers of moles of the substances A and B.

However, if the rate is measured as $\mathrm{mol} / \mathrm{cc} \mathrm{s}$ and $\mathrm{V}$ (the volume) is constant, so $\mathrm{w}$ will be at $\mathrm{v}<<\mathrm{c}$ :

$$
w=-\frac{1}{v_{\mathrm{A}}} \frac{d([\mathrm{~A}] / V)}{d t}=-\frac{1}{v_{\mathrm{A}} V} \frac{d[\mathrm{~A}]}{d t}=\frac{1}{v_{\mathrm{B}} V} \frac{d[\mathrm{~B}]}{d t} .
$$

If the volume is not constant, so we have, e.g., for $w$ of the substance A varying:

$$
w=-\frac{1}{v_{\mathrm{A}}} \frac{d([\mathrm{~A}] / V)}{d t}=-\frac{1}{v_{\mathrm{A}} V} \frac{d[\mathrm{~A}]}{d t}+\frac{[\mathrm{A}]}{v_{\mathrm{A}} V} \frac{d \ln V}{d t} .
$$

If the substance content varies from one point to another of the system under study, we shall have for $w$ :

$$
w \sim \frac{\partial^{2} n_{i}}{\partial t \partial V}=\frac{\partial\left(\partial n_{i} / \partial V\right)}{\partial t}=\frac{\partial \rho_{i}(x, y, z)}{\partial t}
$$

where $n_{i}$ is a substance " $i$ " mole number $(i=\mathrm{A}, \mathrm{B}, \ldots$, and so on), $\rho_{i}(x, y, z)$ is the density of the substance " $i$ " at the point $x, y, z(\mathrm{~mol} / \mathrm{cc})$.

Now let us have a chemical reaction of the type:

$$
v_{\mathrm{A}} \mathrm{A}+v_{\mathrm{B}} \mathrm{B} \Leftrightarrow v_{C} \mathrm{C}+v_{D} \mathrm{D}
$$

for example,

$$
2 \mathrm{Na}+2 \mathrm{H}_{2} \mathrm{O} \Leftrightarrow 2 \mathrm{NaOH}+\mathrm{H}_{2}
$$

then we have for $\mathrm{w}(\mathrm{mol} / \mathrm{s})$ :

$$
w=-\frac{1}{v_{\mathrm{A}}} \frac{d[\mathrm{~A}]}{d t}=\frac{1}{v_{C}} \frac{d[C]}{d t}
$$

and so on.

The formulae (3) - (6), (9) are the definitions of w; there are dependences connecting this rate with some parameters, i.e., $w=w\left(T, t, V, n_{i}, p \ldots\right)$. Of course, $\mathrm{w}$ and $\mathrm{w}(\mathrm{T}, \mathrm{t}, \ldots)$ must transform identically as $v \rightarrow c$. Then using this identity, we 
may get opportunity to solve our problems. But in order to show up clearly this opportunity, we are to concern the problems of the relativistic thermodynamics, using, in particular, results obtained in Ref.1.

According to M.Planck ${ }^{2}$, the first and second principles of thermodynamics are conserved at any velocities of motion of the system (of course, we are studying only inertial frames of references) i.e.,

$$
\begin{gathered}
d Q=d U-d A, \\
d S=d Q / T, S=S_{0}, \\
H=\frac{H_{0}}{\sqrt{1-\beta^{2}}},
\end{gathered}
$$

and

$$
\begin{gathered}
T=T_{0} \sqrt{1-\beta^{2}} ; \beta=\frac{v}{c}, \\
p=p_{0},
\end{gathered}
$$

where $Q$ is the heat put into the system under study or carried off it $(\mathrm{J}), \mathrm{U}$ is the internal energy $(\mathrm{J}), A$ is the work done by the system or with it, $S$ is the entropy of a moving object, and $S_{0}$ is the entropy of the object at rest (there and in the following the subscript " 0 " denotes a quantity in the frame of reference in which the object under study is at rest), $H\left(H_{0}\right)$ is the enthalpy; $\mathrm{c}$ is the velocity of light, $p\left(p_{0}\right)$ is the pressure, $T\left(T_{0}\right)$ is the absolute temperature.

$$
d A=-p d V+\mathbf{v} \cdot d \mathbf{G}
$$

where $\mathbf{G}$ is the momentum, the symbol "•" means vector multiplication.

$$
\begin{gathered}
\mathbf{G}=\mathbf{v} \frac{U_{0}+p_{0} V_{0}}{c^{2} \sqrt{1-\beta^{2}}} \\
V=V_{0} \sqrt{1-\beta^{2}}
\end{gathered}
$$

$\mathrm{G}$ and $\frac{i}{c}(U+p V)$ form the four dimensional vector (4vector) of energy-momentum, which has an invariant length equal to

$$
\frac{i}{c}\left(U_{0}+p_{0} V_{0}\right)
$$

where $i$ is imaginary unity.

But, as it was shown by Veitsman ${ }^{1}$, the absolute temperature $T$ must be varying under relativistic conditions according to X.Ott ${ }^{3}$, i.e.,

$$
T=T_{0} / \sqrt{1-\beta^{2}}
$$

Here we must add as well that the second thermodynamics principle, i.e., (11) we can write down, e.g., in the fol- lowing form when $v \approx c$ :

$$
\delta S=\frac{\delta Q}{S p T^{\alpha \beta} / 3}=\frac{\delta Q}{T_{\alpha}^{\alpha} / 3}
$$

where $T^{\alpha \beta}$ is the temperature tensor of rank 2, $\left(T_{\alpha}^{\alpha} / 3\right)$ is its module.

We have a right to represent the temperature as a tensor of rank 2 according to following reasons. Indeed, let us have an observer at rest in a laboratory reference frame and an object is moving with a velocity $\mathrm{v} \rightarrow \mathrm{c}$. The velocity $\mathbf{v}$ is parallel to coordinate axes $X_{1}$ and $X_{1}^{\prime}$ (see Fig.1).

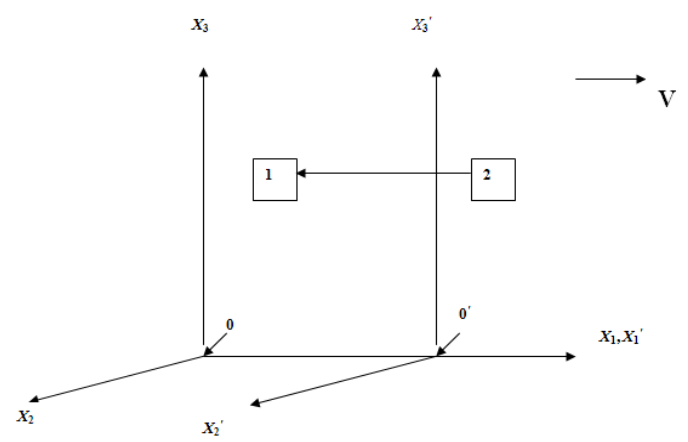

Fig. 1. Two reference frames: $X_{1}, X_{2}, X_{3}$ (at rest) and $X_{1}{ }^{\prime}, X_{2}{ }^{\prime}, X_{3}{ }^{\prime}$ (in moving). 1 is an observer; 2 is an object under study, where chemical reaction is proceeding. $V$ is the velocity of the second reference one.

The velocity components of the molecules (the atoms) within the object will be equal for observer (according to Special Relativity) to:

$$
\begin{gathered}
w_{1}=\frac{w_{1}^{\prime}+v}{1+\frac{v w_{1}^{\prime}}{c^{2}}}, \\
w_{2}=\frac{w_{2}^{\prime} \sqrt{1-\beta^{2}}}{1+\frac{v w_{1}^{\prime}}{c^{2}}}, \\
w_{3}=\frac{w_{3}^{\prime} \sqrt{1-\beta^{2}}}{1+\frac{v w_{1}^{\prime}}{c^{2}}},
\end{gathered}
$$

where $w$ is an object velocity (of molecule, atom) in a laboratory reference frame; $w_{1}, w_{2}, w_{3}$ are its components; $w^{\prime}$ is an object velocity for an observer being in the moving reference frame; $w_{1}^{\prime}, w_{2}^{\prime}, w_{3}^{\prime}$ are its components.

As seen, $w_{2}=w_{3} \neq w_{1}$ as $v \rightarrow c$ but the temperature of gas or liquid depends on the velocities of their microprticles relative the object mass centre. Then we can represent the temperature as $T^{\alpha \beta}$ when $v \rightarrow c$ and $T g^{\alpha \beta}$ if $\mathrm{v}<<\mathrm{c}$ where $g^{\alpha \beta}$ is the fundamental tensor (3D-formalism), i.e., 


$$
g^{\alpha \beta}=\left(\begin{array}{lll}
1 & 0 & 0 \\
0 & 1 & 0 \\
0 & 0 & 1
\end{array}\right), \alpha, \beta=1,2,3 .
$$

then $T=\frac{1}{3} T_{\alpha}^{\alpha}$ is a module of the temperature tensor, i.e. the same temperature $T$, which we know from thermodynamics.

Now we see that our chemical reaction problems are closely allied with the relativistic thermodynamics (and, as we shall see later, with irreversible thermodynamics) as $v \rightarrow c$. Therefore the main goal of this article cannot be overcome without taking into account the problems of the relativistic one.

\section{The Chemical Reaction Rate as $v \rightarrow c$}

There are two ways to solve the problem: first, using the law of mass action; second, within the framework of irreversible thermodynamics. Using the law of mass action is connected with some complexity that today we can hardly solve. Indeed, we can write, e.g., for the reaction (7):

$$
w=k[\mathrm{~A}]^{v_{\mathrm{A}}}[\mathrm{B}]^{v_{\mathrm{B}}},
$$

where $k$ is a reaction rate coefficient (the forward direction).

Theoretically the coefficient is found by the methods of the quantum chemistry. Now it is determined only for the simplest case at $v<<c$. Therefore for solving our problem we cannot be using the mass action law but we can use the irreversible thermodynamics (see, e.g., Ref.4).

Let us have any macroscopic size imaginary reactor, which is moving with the velocity $v \rightarrow c$ and where the reaction of type (1) is running (see Fig 1). The reaction is near the equilibrium. Then the reaction rate will be for an observer being in the laboratory reference frame (i.e., at rest; $\mathrm{mol} / \mathrm{s})$ :

$$
w=-\frac{1}{v_{\mathrm{A}}} \frac{d[\mathrm{~A}]}{d t}=a A_{r}=(a R T) \frac{A_{r}}{R T}=a_{1} A_{1},
$$

where $a$ is a phenomenological coefficient, $A_{r}$ is the affinity according to De Donder.

$$
A_{r}=+v_{\mathrm{A}} \mu_{\mathrm{A}}-v_{\mathrm{B}} \mu_{\mathrm{B}}=-\sum_{i} v_{i} \mu_{i} ; i=\mathrm{A}, \mathrm{B}
$$

where $\mu_{i}$ is the chemical potential of the substances $\mathrm{A}$ and $\mathrm{B}(\mathrm{J} / \mathrm{mol})$.

The quantity $a_{1}$ is the rate of the chemical reaction ( $\left.\mathrm{mol} / \mathrm{s}\right)$ in the forward direction.

As $v \rightarrow c$,

$$
t=t_{0} / \sqrt{1-\beta^{2}}
$$

but $\mathrm{A}=\mathrm{A}_{0}$, and

$$
w=w_{0} \sqrt{1-\beta^{2}}=-\frac{1}{v_{\mathrm{A}}} \frac{d\left[\mathrm{~A}_{0}\right]}{d t_{0}} \sqrt{1-\beta^{2}}
$$

So as $v \rightarrow c$ the quantity $w \rightarrow 0(\mathrm{~mol} / \mathrm{s})$.

Under the relativistic conditions the quantity $a_{1}$ must transform like $w$, i.e., $a_{1}=a_{01} \sqrt{1-\beta^{2}}$, but at that time the quantity $A_{1}$ must be a relativistic invariant, namely $A_{1}=A_{01}$. Taking into consideration (19) or (20), we shall show that $A_{1}$ is the relativistic one.

Taking (15) into consideration as well, we can represent the chemical potential $\mu_{i}$ of the substance " $i$ " as (see Ref.[4]):

$$
\mu_{i}=\left(\frac{\partial H}{\partial n_{i}}\right)_{S, V, n_{j}, v}, i \neq j
$$

The expression (30) is obtained from the following dependence (see again Ref.[4] and (15)):

$$
d H=T d S+V d p+\mathbf{v} \cdot d \mathrm{G}+\sum_{i} \mu_{i} d n_{i}
$$

and taking into consideration (12) and (19) or (20), we can see that the quantity $A_{1}$ is indeed the relativistic invariant.

If we define $\mathrm{w}$ as $\mathrm{g} / \mathrm{s}$ or $\mathrm{mol} / \mathrm{cc} \mathrm{s}$, so it and a1 will not vary as $v \rightarrow c$ since $\mathrm{V}$ will vary according to (17) but the substance mass $m$ according to the well-known formula

$$
m=m_{0} / \sqrt{1-\beta^{2}}
$$

If we define $\mathrm{w}$ as $\mathrm{g} / \mathrm{cc} \cdot \mathrm{s}$, so as $v \rightarrow c$ it and al will be tending to $\infty$, but $w$ will be near the equilibrium since the number of reacting microparticles (molecules, atoms) does not increase; their masses grow (see Discussion as well).

So the chemical reaction rate depends on its definition, i.e., on a dimensional representation chosen.

If the substance density varies from one point to another of the system under study (see (6) ), so

$$
w_{i}(x, y, z)=\frac{\partial \rho_{i}(x, y, z)}{\partial t}=a_{1} A_{r}(x, y, z) / R T
$$

and $A_{r}(x, y, z) / R T$ will be the Lorentz-invariant as well. We shall show it.

Let us take the following relationship (see (31)):

$$
d H_{V}=T d S_{V}+d p+\mathrm{v} \cdot \mathrm{dGv}+\sum_{i} \mu_{i} d \rho_{i}
$$

here the subscript $V$ means that a quantity is divided into volume $\mathrm{V}$, i.e., it (this quantity) is a specific one.

From (34) we have

$$
\mu_{i}(x, y, z)=\left[\partial H_{V} / \partial \rho_{i}\right]_{S_{V}, p, \rho_{i}, v} ; i \neq j
$$

since $H_{V}=\delta H / \delta V, \rho_{i}(x, y, z)=\delta m_{i} / \delta V$, and so here

$$
\mu_{i}(x, y, z) \sim 1 / \sqrt{1-\beta^{2}},
$$


Taking into consideration (31), we can see that $A_{r}(x, y, z) / R T$ or $3 A_{r}(x, y, z) / R T_{\alpha}^{\alpha}$ will the Lorentz-invariant only if $\mathrm{T}\left(T_{\alpha}^{\alpha}\right)$ will be varying according to X.Ott, i.e., according to (19) or (20). If we take the temperature transformation according to M.Planck as $v \rightarrow c$ (i.e., according to (13)) or according to H.Callen and G.Horwitz [5] (i.e. $T=T_{0}$ ), so we shall come to absurdity. Indeed, in these cases the left and the right sides (34) will transform in different ways under relativistic conditions.

\section{The State Equation for Perfect Gases under Relativistic Conditions and the Relativistic Temperature}

As known, the temperature is a very important thermodynamic parameter under studying the rate of the chemical reactions. As known as well, the large number of those is going on within gaseous phase where, as a rule, it is simpler to be studying the processes of the chemical transformations. Therefore we can obtain important information on the relativistic temperature studying just the relativistic thermodynamics of perfect gases. But first of all we shall concern here works where their authors make attempts to deal with the temperature as a vector, e.g., in the following form (so called the inverse $T 4$-vector) (see Ref. 6, 7):

$$
\beta^{\mu}=\frac{v^{\mu}}{k T} ; \mu=0,1,2,3
$$

where $v^{\mu}$ is a 4-velocity vector.

Taking into consideration (21) - (23), we can do the following conclusion. The average magnitudes of the microparticle velocity components within the studying object will not be equal to one another as $v \rightarrow c$, i.e., in this case we can represent the temperature as a tensor of rank 2 . What is more, we can represent $T$ either as a tensor of rank 2 or as a scalar when $v<<c$. On the contrary, we cannot represent the inverse $T$ vector as a scalar under these conditions since any vector can never be reduced to scalar one way or another. Below we shall obtain the state equation for perfect gases in 4D-formalism under relativistic conditions when the temperature of the system either the scalar or the tensor of rank 2 but it (the temperature) is not any vector.

We consider that our system can be characterized by a 4D-energy tensor $\Pi^{\alpha \beta}$ of rank 2 .

If $\Pi^{\alpha \beta}$ depends on $\bar{v}$ which is an average magnitude of the absolute value of the micropartile velocity $v$ relative to the mass centre, then we shall have for the 4-tensor components (perfect gases):

$$
\Pi^{\alpha \beta}=\left(\begin{array}{cccc}
\Pi^{00} & 0 & 0 & 0 \\
0 & \Pi^{11} & 0 & 0 \\
0 & 0 & \Pi^{22} & 0 \\
0 & 0 & 0 & \Pi^{33}
\end{array}\right),
$$

$$
\begin{aligned}
& \Pi^{00}=\frac{\mu_{0} c^{2}}{1-\left(\frac{\bar{v}}{c}\right)^{2}} ; \\
& \Pi^{11}=\frac{\mu_{0}{\overline{v^{1}}}^{2}}{1-\left(\frac{\bar{v}}{c}\right)^{2}}, \\
& \Pi^{22}=\frac{\mu_{0}{\overline{v^{2}}}^{2}}{1-\left(\frac{\bar{v}}{c}\right)^{2}}, \\
& \Pi^{33}=\frac{\mu_{0}{\overline{v^{3}}}^{2}}{1-\left(\frac{\bar{v}}{c}\right)^{2}},
\end{aligned}
$$

where $\mu_{0}=\frac{m_{0}}{V_{0}}=\frac{\delta m_{0}}{\delta V_{0}} ; \overline{v^{1}}, \overline{v^{2}}, \overline{v^{3}}$ are the components of $\overline{v^{\alpha}} ; \Pi^{11}+\Pi^{22}+\Pi^{33}=p_{0}=\frac{1}{3} p_{0} \delta_{. \alpha}^{\alpha} ; \alpha=1,2,3$ if $\bar{v}<<c ;$ $\delta_{\alpha}^{\beta}$ is Kronecker's object.

There are expressions of the form $\frac{{\overline{\alpha^{\alpha}}}^{2}}{1-\beta^{2}}$ and an expression of the form $\frac{c^{2}}{1-\beta^{2}}$ in (39). Those are component squares of the 4-velocity.

$$
\begin{gathered}
S p \Pi^{\alpha \beta}=\Pi_{\alpha}^{\alpha}=\Pi^{11}+\Pi^{22}+\Pi^{33}-\Pi^{00}=-\mu_{0} c^{2}=\text { in var } . \\
\Pi^{11}+\Pi^{22}+\Pi^{33}=\Pi^{00}-\mu_{0} c^{2}=\frac{M_{0} c^{2}}{V_{0}\left(1-\frac{\bar{v}^{2}}{c^{2}}\right)}-\frac{M_{0}}{V_{0}} c^{2}= \\
\left(\frac{M_{0}}{V_{0}}\right) \frac{c^{2}-c^{2}+\bar{v}^{2}}{1-\frac{v^{2}}{c^{2}}}=\frac{M_{0} \bar{v}^{-2}}{\sqrt{1-\frac{v^{2}}{c^{2}}}} \\
V\left(\Pi^{11}+\Pi^{22}+\Pi^{33}\right)=\frac{M_{0} \bar{v}^{2}}{\sqrt{1-\frac{v^{2}}{c^{2}}}} \approx \bar{E}
\end{gathered}
$$

since $\bar{E}$ depends on (see Ref.8)

$$
\sqrt{\overline{v^{2}}}=1,22\left(\frac{2 k T}{m}\right)^{1 / 2}
$$

but

$$
\bar{v}=1,13\left(\frac{2 k T}{m}\right)^{1 / 2}
$$

and 


$$
\frac{p_{0} V}{1-\frac{\bar{v}^{2}}{c^{2}}} \approx \frac{3 N k T}{2}
$$

Since $V=V_{0} \sqrt{1-\beta^{2}}$, so $T=T_{0} / \sqrt{1-\frac{\bar{v}^{2}}{c^{2}}}$ in almost full agreement with (42) and (46) (see below).

In (42) and (46) we have taken into account that the average energy $\bar{E}$ (for the perfect gases) is equal to (Ref.2):

$$
\bar{E}=\frac{3}{2} N k T \text {. }
$$

Equation (45) is akin to the equation of state for the perfect gases under relativistic conditions:

$$
\frac{p V}{1-\beta^{2}}=N k T
$$

If $v=0$, (47) becomes the well-known equation of state for perfect gases, i.e.,

$$
p V=N k T,
$$

but (45) has coefficient $3 / 2$ on its right side. It appeared for the following reason. In (47) the term $N k T$ is related to the most probable microparticle velocity $v_{p r}$; in (45) the term $\frac{3}{2} N k T$ is related to the root-mean-square velocities $\sqrt{\overline{v^{2}}}$ of gas molecules (atoms). As known, $\sqrt{\overline{v^{2}}}=\sqrt{\frac{3}{2} v_{p r}}$.

Compare the well known formula

$$
E=\frac{m_{0} c^{2}}{\sqrt{1-\beta^{2}}} .
$$

with (46). Evidently the temperature $T$ can vary in them only according to (19) as $v \rightarrow c$. Compare (45) and (47). The dependence (47) was obtained under the conditions that $T$ varies according to (19) and the pressure $p$ is Lorentz-invariant, i.e., $p=p_{0}$. As seen from (45), we can consider it twofold. Either ${ }^{p=\frac{p_{0}}{1-\frac{v^{2}}{c^{2}}}}$ in Minkowski space or again $p=p_{0}$ in it but $\left(1-\frac{\bar{v}^{2}}{c^{2}}\right)^{-1}$ is a coefficient in the left side (45). The same dependence (45) will be also obtained in another reference frame, e.g., moving with a velocity $\mathrm{v}$ along the axis $X_{1}$.

\section{Discussion and Conclusions}

There arises a question: if these physical-chemical constructions result in violation of the causality principle. In our opinion,"No!" We shall show it using the following example.

Let an observer, co-moving a reference frame as $v \rightarrow c$, contemplate to observe chemical reaction (2) at the temperature $T_{01}$. Being in a laboratory reference frame, another observer contemplates to study this process as well. Evidently, the temperature $T$ and the reaction heat $\mathrm{Q}$ in the object under study will be equal to $T=T_{0} / \sqrt{1-\frac{v^{2}}{c^{2}}}$ and $Q=Q_{0} / \sqrt{1-\frac{v^{2}}{c^{2}}}$ for the second observer, where $T_{0}$ and $Q_{0}$ are the proper temperature and reaction heat of the above object. $T$ can be much higher than $T_{01}$. Let the substance A transform itself fully into the substance B at the temperature $T$. Will the causality principle then be violated? Indeed, the observer in the laboratory reference frame will be allowed to see only the substance which is already disintegrating, whereas the observer in moving reference frame will see the substance, which is not decaying any more. But such situation will not take place since the beginning of the substance A full decay must take place for the observer in the laboratory reference frame at temperature $T_{1}=T_{01} / \sqrt{1-\beta^{2}}$, which will exceed $T$, i.e., we must take into consideration variations of different parameters affecting characteristic properties of the thermodynamical (chemical) process under relativistic conditions. Therefore, if, e.g., reaction (1) is near its equilibrium for the observer co-moving with the reference frame, so it will be near the equilibrium and for the observer being in the laboratory reference frame. The pattern of the process is to be qualitatively identical for the both observers otherwise the causality principle will be violated.

In the and of the article we can make the following main conclusions.

The rate of the chemical reaction as $v \rightarrow c$ depends in particular on its definition. Solving problems of quantum chemistry under relativistic conditions, for example, studying how $k$, the coefficient of reaction rate, will depend on $\mathbf{v}$, we must take into account the law of the temperature varying at the relativistic ones. The law states $T=T_{0} / \sqrt{1-\beta^{2}}$ or $T=\frac{1}{3} T_{\alpha}^{\alpha}=T_{0} / \sqrt{1-\beta^{2}}$, i.e., according to X.Ott.

\section{References}

[1] E.V.Veitsman. J. Experimental and Theoretical Phys. (Russia), 105, 1057 (2007)

[2] M. Planck. Annalen der Physik, 26, 1 (1908).

[3] X. Ott. Zeitschrift f. Phys., 175, 70 (1963).

[4] R. Haase. Thermodynamik des irreversible Prozesse, das Kapitel 1, (Dietrich Steinkopf Verlag, Darmstadt, 1963).

[5] H.Callen and G.Horwitz. Am. J. Phys., 39, 938 (1971).

[6] Victor H.Hamity. Relativistic Thermodynamics, Phys. Rev.,187, 1745 (1969).

[7] Wu, Zhong Chao. Inverse temperature 4-vector in special relativity, Europhys. Lett., 88, 2005/1 - 2005/4 (2009).

[8] V.G.Levich. The Course of Theoretical Physics, vol.1, Part 3, Ch. 2 and 8 (Fizmatgiz, Moscow, 1962), [in Russian]. 